\title{
Perceptual dimensions of tactile surface texture: A multidimensional scaling analysis
}

\author{
MARK HOLLINS, RICHARD FALDOWSKI, SUMAN RAO, and FORREST YOUNG \\ University of North Carolina, Chapel Hill, North Carolina
}

\begin{abstract}
The purpose of this study was to examine the subjective dimensionality of tactile surface texture perception. Seventeen tactile stimuli, such as wood, sandpaper, and velvet, were moved across the index finger of the subject, who sorted them into categories on the basis of perceived similarity. Multidimensional scaling (MDS) techniques were then used to position the stimuli in a perceptual space on the basis of combined data of 20 subjects. A three-dimensional space was judged to give a satisfactory representation of the data. Subjects' ratings of each stimulus on five scales representing putative dimensions of perceived surface texture were then fitted by regression analysis into the MDS space. Roughness-smoothness and hardness-softness were found to be robust and orthogonal dimensions; the third dimension did not correspond closely with any of the rating scales used, but post hoc inspection of the data suggested that it may reflect the compressional elasticity ("springiness") of the surface.
\end{abstract}

A complex sensory experience occurs when a person draws a finger across the surface of an object. In addition to whatever information may be gained about the shape and other geometrical properties of the object, the observer also receives impressions related to the nature of the surface. These experiences of surface texture are the subject of this report. Although some aspects of surface texture have been extensively studied, an overall understanding of texture perception remains elusive. In the words of Connor, Hsiao, Phillips, and Johnson (1990), "Tactile texture perception is poorly understood; with few exceptions, little is known about its dimensionality, its physical determinants, or its neural mechanisms."

Modern study of texture perception dates from the work of David Katz, whose monograph The World of Touch $(1925 / 1989)$ set the agenda for much of the later work on the subject. Katz asked subjects to discriminate, and to describe, a wide variety of tactile surfaces under various conditions of touching. One of Katz's central concepts was a distinction between two types of surface properties, which he called Modifikationen (qualities) and Spezifikationen (identifying characteristics). By "qualities," he meant properties on which any tactile surface could be rated; he mentioned roughness and hardness as two such dimensional qualities, but left open the possibility that there were others, as yet unidentified. By "identifying characteristics," Katz meant the characteristic overall feel of a surface-the "leatheriness" of leather, the "rubberiness" of rubber, and so on.

The authors are indebted to Mary McFarlane and Chris Wiesen for expert statistical advice. S. Rao is now affiliated with the Department of Psychology, 102 Gilmer Hall, University of Virginia, Charlottesville, VA 22903 . Reprint requests should be addressed to $M$. Hoilins, Department of Psychology, CB 3270, Davie Hall, University of North Carolina, Chapel Hill, NC 27599.
It is unclear in Katz's writings, probably because he had no settled opinion on the subject, exactly what the relationship is between these two types of properties. Are identifying characteristics simply certain combinations of values of the qualities? That is, will all surfaces with a particular roughness, combined with a particular hardness and particular values on other qualitative dimensions that may exist, feel like the same material? Or, alternatively, does the identifiable feel of a surface depend on aspects that are not captured by any scalable qualities?

A somewhat more general way to phrase these questions is to ask whether perceptions of surface texture are interrelated in a way that can be captured by a perceptual space, and if so, whether the dimensions of this space are meaningful tactile qualities such as roughness and hardness. These are the questions addressed in the present study, with the use of a two-step experimental design. First, multidimensional scaling (MDS) is used to organize tactile surfaces into a perceptual space where the distance between two objects reflects their perceptual differentness; stress values and other properties of the MDS solutions are examined to determine the reasonableness of describing apparent texture in terms of such a space. Second, the ability of roughness, hardness, and other putative qualities to describe this space-as well as the independence of their contributions to it-is then evaluated.

This second step will help not only in the evaluation of the usefulness of the MDS space, but also in the attempt to achieve an increased understanding of the dimensional qualities of tactile surface texture perception. There is no doubt that roughness has the psychometric properties expected of a continuous dimension (Ekman, Hosman, \& Lindstrom, 1965; Lederman \& Taylor, 1972; Stevens \& Harris, 1962), and hardness, although less thoroughly studied, also appears to be a bona fide dimension (Klatzky, 
Lederman, \& Reed, 1987, 1989). Beyond that, however, little is known. Frictional resistance is sometimes perceptible, but the nature of its contribution to sensory experience is not entirely clear (Green, 1981; Lederman, 1978a, 1978b; Taylor \& Lederman, 1975). By examining the way in which textures are distributed in a perceptual space, it may be possible to gain some insight into the existence of tactile dimensions that are not currently recognized as such-that is, into aspects of the sensory experience that are not generally thought of as dimensions.

While much has been learned about tactile dimensions, especially roughness, through the use of ordered sets of stimuli that vary along a well-specified physical continuum, in the present study we make use of more naturalistic stimuli that vary along numerous physical dimensions. An evaluation of the perceptual importance of roughness, hardness, and other putative dimensions of texture requires a determination of whether subjects spontaneously use these dimensions in categorizing everyday surfaces, and whether they can reliably order such stimuli along these dimensions.

The goals of the present study, then, are to determine whether subjects' categorizations of tactile surfaces on the basis of texture can reasonably be described in terms of a "perceptual space" derived through MDS techniques, and if so, to begin identifying the dimensions of this space.

\section{GENERAL METHOD}

\section{Stimuli}

The stimuli were 17 tactile surfaces, chosen to give a wide range of subjective impressions. Seven were thin or flexible materials that were mounted with two-sided tape on blocks of wood measuring $4 \times 4 \times 2 \mathrm{~cm}$. These materials were (1) wax paper; (2) cardboard; (3) smooth plastic; (4) nylon scouring pad; (5) 220-grit sandpaper; (6) felt; and (7) velvet. An additional surface, (8) woven straw, could not be adequately secured to the wood with tape, and so was tacked to it; a longer $(8-\mathrm{cm})$ block of wood was used, and the tacks were applied near its two ends, so that the central region, used as a stimulus, was free of tacks. The other 9 stimuli were the surfaces of, or were parts of, rigid objects, and therefore did not require mounting. These were (9) the cork surface of a coaster; (10) a large rubber eraser; (11) a block of styrofoam; (12) a brick; (13) a block of glossy painted wood, identical to those on which Stimuli 1-7 were mounted (children's building blocks); (14) a (dry) cellulose kitchen sponge; (15) a smooth leather wallet; (16) the internal surface of pine bark; and (17) an unglazed ceramic tile.

All stimulus surfaces were essentially free of overall curvature, except the bark. This was carefully aligned before presentation so that it moved along the subject's fingertip in a direction parallel to the axis of curvature.

\section{Procedure}

The same method of stimulus presentation was used throughout the study. Each subject was tested individually. The subject sat at a table, with his/her right arm extended forward and resting on a pillow. The hand extended beyond the pillow, palm downward, with the wrist moderately flexed so that the extended index finger pointed diagonally downward at an angle of about $45^{\circ}$. The subject made a fist with the other fingers, and wore over them a sock with a hole for the index finger.

The experimenter positioned a stimulus surface beneath the index fingertip and then raised the stimulus, in a direction normal to the surface, so that it made contact with the skin. Enough force was exerted by the experimenter to just visibly displace the index finger upward. The experimenter then drew the stimulus, in a direction perpendicular to its surface, from proximal to distal along the fingertip, for a distance of approximately $2.5 \mathrm{~cm}$, at an estimated speed of $5 \mathrm{~cm} / \mathrm{sec}$. The stimulus was then lowered by the experimenter, until it left the skin. The subject was then asked to give a verbal response, the nature of which differed from one phase of the study to another (see following sections).

An opaque curtain, suspended above the table and perpendicular to the subject's arm at the elbow, blocked his/her view of the hand, stimuli, and experimenter.

Throughout a run, a random noise source (an FM radio set between stations) was present on a nearby table. It was set loud enough to prevent the subject from hearing the faint sounds made by skin contact with the stimuli, but not loud enough to impede verbal communication with the experimenter.

\section{PILOT EXPERIMENT}

The purpose of the pilot experiment was to learn how subjects would describe the stimuli when no guidelines or constraints (other than a request for adjectives) were provided by the experimenters. It was hoped that this would yield some insight into the identity of salient perceptual dimensions of surface texture, and would also provide a list of adjectives from which could be drawn a subset for use in the main experiment.

\section{Method}

Subjects. The subjects in the pilot experiment were graduate and undergraduate students ( 3 males, 2 females), recruited informally. They ranged in age from 20 to 24 . All were right-handed, as assessed by observation of the hand used for writing, and by verbal report.

Procedure. On each trial, a stimulus was presented, and the subject was asked to give one or more descriptors for it. The experimenter explained that adjectives, rather than the names of objects, were particularly desired. A stimulus was frequently presented more than once, at the request of the subject. When the subject indicated that he/she had no further descriptors to offer for one stimulus, the experimenter presented the next stimulus in the series. A different random order of the stimuli was used for each subject.

\section{Results}

Subjects differed somewhat in number of descriptors offered, from 1.6 to 3.8 descriptors per stimulus. All 5 subjects used the words smooth and rough; 4 used soft (or softer), but only 2 used hard (1 subject used neither term). Bumpy, coarse, flat, fuzzy, grainy, and slick were among the other adjectives used by more than 1 subject. Regarding temperature sensations, 2 subjects used cold and a 3rd, cool; none used warm. Three of the subjects remarked on the seeming absence of movement in the presentation of the smooth plastic surface; their impression was that it was held stationary throughout its period of contact with the skin. For some stimuli, there was fair consistency of response from one subject to the next, as is illustrated by their descriptions of cardboard: "smooth, wood, grainy, polished, sanded"; "slick"; "slick, smooth, hard"; "slick, [with] raised areas"; "smooth, chalky, powdery." Other stimuli, however, evoked more 
diverse responses. The eraser, for example, was variously described as "steel, cold, unfinished steel, durable"; "cold, velvety"; "smooth, hard"; "slick"; and "grainy."

\section{MAIN EXPERIMENT}

The main experiment consisted of two phases. The purpose of the first phase was to obtain data indicating the perceived dissimilarity of the stimulus surfaces from one another. MDS procedures were then used to construct a model of perceptual space on the basis of these dissimilarities. Because the model constructed by MDS was based solely on subjects' similarity groupings of tactile sensations, the positions of stimuli within this space were free from any constraining or contaminating influence exerted by the semantic content of experimenter-selected rating scales.

In the second phase, the stimuli were rated on a set of adjective scales, in order to determine whether these scales provided a useful way of interpreting the model of perceptual space. It should be noted that there were no a priori guarantees that these particular adjectival rating scalesselected on the basis of plausibility, tradition, and the results of the preliminary experiment, rather than by means of a systematic exploration of the semantics of tactile surface texture perception--would correspond to the dimensions of the MDS model in any simple way.

\section{Method}

Subjects. The subjects in the main experiment were introductory psychology students, who participated in order to fulfill a course requirement. There were 10 males and 10 females, ranging in age from 18 to 22 . All were right-handed.

Procedure. In Phase 1, the experimenter asked the subject to assign the stimuli to groups. When the first stimulus was presented, the experimenter said, "This is Stimulus 1. Call the group it belongs in, Group A." Presenting the next stimulus, identified to the subject as Stimulus 2, the experimenter told the subject to assign it also to Group A if it felt "fairly similar" to Stimulus 1, or, alternatively, to assign it to Group B if it felt "fairly different" from Stimulus 1. The experimenter proceeded in this way to present all 17 stimuli, using a different random order for each subject. The subjects were told at the outset that they had to create at least three groups, but could not create more than seven; no restrictions were placed on the number of objects that could be placed in a group, however. A given stimulus could, of course, be assigned to only one group. The subjects were required to use single, consecutive letters, not descriptive terms, in referring to the groups. At the request of the subject, a given stimulus was often presented more than once; in addition, the subjects were allowed, and in fact encouraged, to review previously presented stimuli and to revise the groupings if appropriate. There were no constraints, other than the 3-7 limit on groups, on such rearrangements; groups could be combined or subdivided, and individual stimuli could be switched from one group to another. This phase of the experiment continued until the subject, following a final review of all stimuli, presented by group, declared himself/herself satisfied with the way in which they were grouped.

Each subject participated in Phase 2 of the experiment immediately after completing Phase 1 . The experimenter presented the same stimuli again, using for each subject the same order that had been followed at the start of Phase 1. As each stimulus was presented, the subject was asked to indicate where it belonged on each of five adjective scales: smosth-rough, hard-soft, slippery-sticky, flatbumpy, and warm-cool. These scales were selected as representing, in the view of the experimenters, most of the descriptive concepts employed (though with terminological variations) by subjects in the preliminary experiment; each of the terms except warm had in fact been used by at least 1 subject. Each adjective pair was presented as a visual analogue scale: a horizontal line $12.8 \mathrm{~cm}$ in length, with one adjective at each end (smooth, hard, slippery, flat, and warm always being on the left). Each scale was on a separate slip of paper. They were passed under the curtain to the subject by the experimenter, one at a time; the subject made a vertical mark on the paper, intersecting the scale at what he/she judged to be the appropriate position, and then passed the paper back to the experimenter before receiving the next scale. The stimulus was presented once (or more, if the subject so requested) in conjunction with each of the scales. All five scales were completed for a given stimulus before the experimenter proceeded to the next stimulus; the scales were presented in a randon order which was independently chosen for each object, for each subject.

\section{Results}

The data from Phase 1 were tallied in the form of a cooccurrence matrix for all stimulus combinations (see Table 1). The co-occurrence of a particular stimulus pair for a given subject was taken as unity if the subject placed those two stimuli in the same group, and as zero if he/she placed them in different groups. The mean co-occurrence value for each stimulus pair was then computed across subjects. The table indicates a large number of stimulus pairs with either high or low co-occurrence values, leading one to anticipate an MDS solution containing a number of clusters or constellations of stimulus objects separated by relatively large distances between clusters.

The mean co-occurrence values were next subtracted from unity to convert the matrix into one of non-cooccurrence, or, by assumption, dissimilarity. These dissimilarity values were then analyzed using the MDS program, ALSCAL 84.1, PC version (Young \& Lewyckyj, 1979), assuming an interval level of measurement. The program was instructed to develop spatial arrangements of the stimuli in $1,2,3,4,5$, and 6 dimensions under the constraint that S-Stress be minimized in each case. ALSCAL experienced numerical convergence difficulties with 1- and 5-dimensional solutions, which we take as an indication that 1 dimension was grossly inadequate for approximating the observed dissimilarities and 5 dimensions may have been insufficiently constrained by dissimilarities derived from only 17 stimuli.

MDS solution dimensionality. Conceptually, ALSCAL attempts to approximate the dissimilarities (non-cooccurrences) between stimuli by interstimulus distances within an MDS space of the desired solution dimensionality. Three measures have been proposed to describe the degree to which MDS solution distances deviate from their corresponding dissimilarities: S-Stress (Takane, Young, \& de Leeuw, 1977), Stress (Kruskal, 1964a, 1964b; Kruskal \& Wish, 1978), and $1-R^{2}$. In short, Stress is defined on distances, S-Stress is defined on squared distances, and $1-R^{2}$ refers to the proportion of variance in the dissimilarities not accounted for by a regression of the dissimilari- 


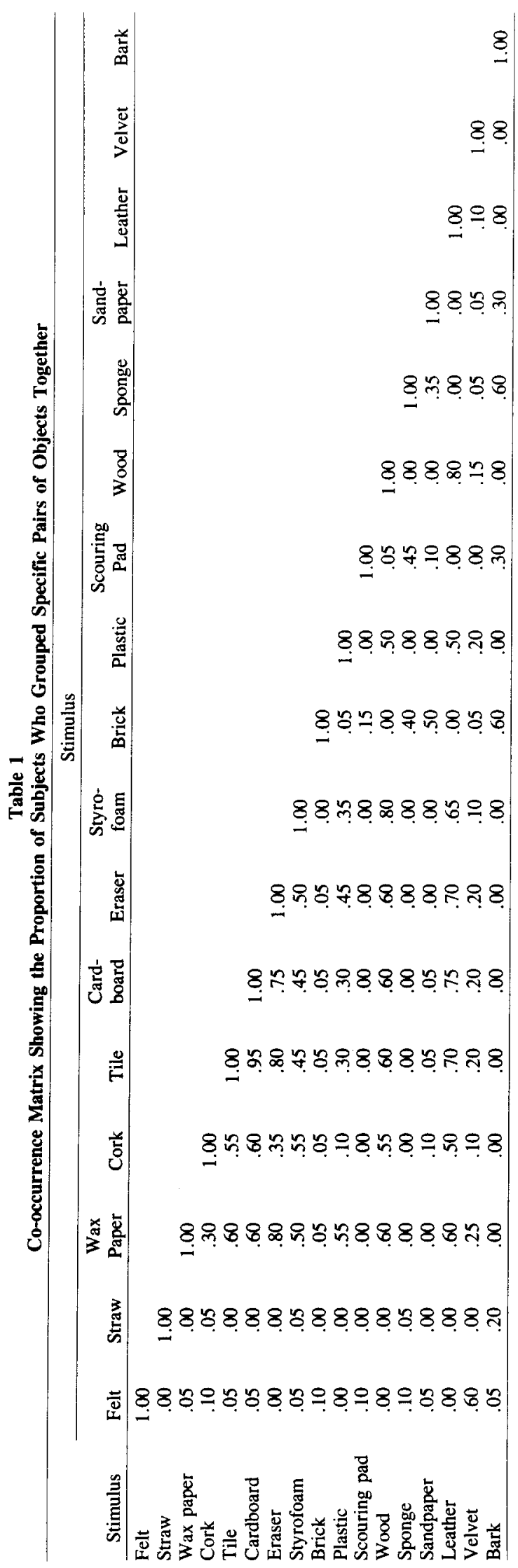

ties onto the MDS distances. Values for each of these measures range from 0 , indicating perfect fit, to 1 , indicating no fit at all. Among the three measures, Young and Lewyckyj (1979) recommend focusing on $1-R^{2}$ because of its straightforward interpretation.

Scree plots (Cattell, 1978) of each measure may heuristically guide an analyst in selecting an appropriate solution dimensionality. According to the scree criteria, the dimensionality at which a sharp "elbow" is seen in the scree curve indicates an appropriate choice of solution dimensionality. As shown in the scree plots of S-Stress, Stress, and $1-R^{2}$ displayed in Figure 1, values of each measure decrease with increasing solution dimensionality; however, no distinctive bends are apparent in the plots for S-Stress and Stress. The plot of $1-R^{2}$, on the other hand, seems to have a slight break at either 3 or 4 dimensions, but the precise choice between the two is not clear.

As another way of comparing the solutions of different dimensionalities, we examined in each case the relation between MDS distances and dissimilarities. The 1- and 2-dimensional MDS solutions reveal inadequacies in which medium to small dissimilarity values (less than about 0.7 ) are represented by uniformly small MDS distances, while larger dissimilarities are represented by small, medium, and large distances in no ostensible pattern. For the 3-dimensional solution, there is a systematic tendency for MDS distance to increase with dissimilarity, which firmly establishes 3 dimensions as the minimum dimensionality of an adequate MDS solution. The relation between MDS distance and dissimilarity is comparable in the 3- and 4-dimensional solutions with regard to medium and small dissimilarities (below about 0.6 ), but for larger dissimilarities, the relation between distance and dissimilarity is closer in the 4-dimensional solution. This modest improvement results in the nominal .04 drop in the value of $1-R^{2}$ between the 3- and 4dimensional MDS solutions.

In contrasting the 3- and 4-dimensional MDS solutions, one might ask how the basic configurations are alike and how they are different. Table 2 shows the scores of the stimuli on each solution dimension for the 3- and 4dimensional cases, while Table 3 presents the matrix of intercorrelations between dimensions of the 3- and 4dimensional solutions. Basically, the high correlations between the first three dimensions of each solution indicate that they are capturing similar aspects of the dissimilarity judgments. (Occurrence of this result is by no means guaranteed in MDS solutions.) Thus, while the fourth dimension of the 4-dimensional solution is correlated with the three dimensions of the 3-dimensional solution to a small degree, it appears primarily to be reflecting an aspect of the dissimilarity judgments either missed or deemphasized in the 3-dimensional solution. Examination of the ordination of the stimuli on the first three solution dimensions for the 3- and 4-dimensional cases confirms that corresponding solution dimensions are similar in their macroscopic patterns. However, perusal of the pattern of ordination on the fourth dimension in the 4-dimensional solution reveals no obvious motif in its configuration; per- 


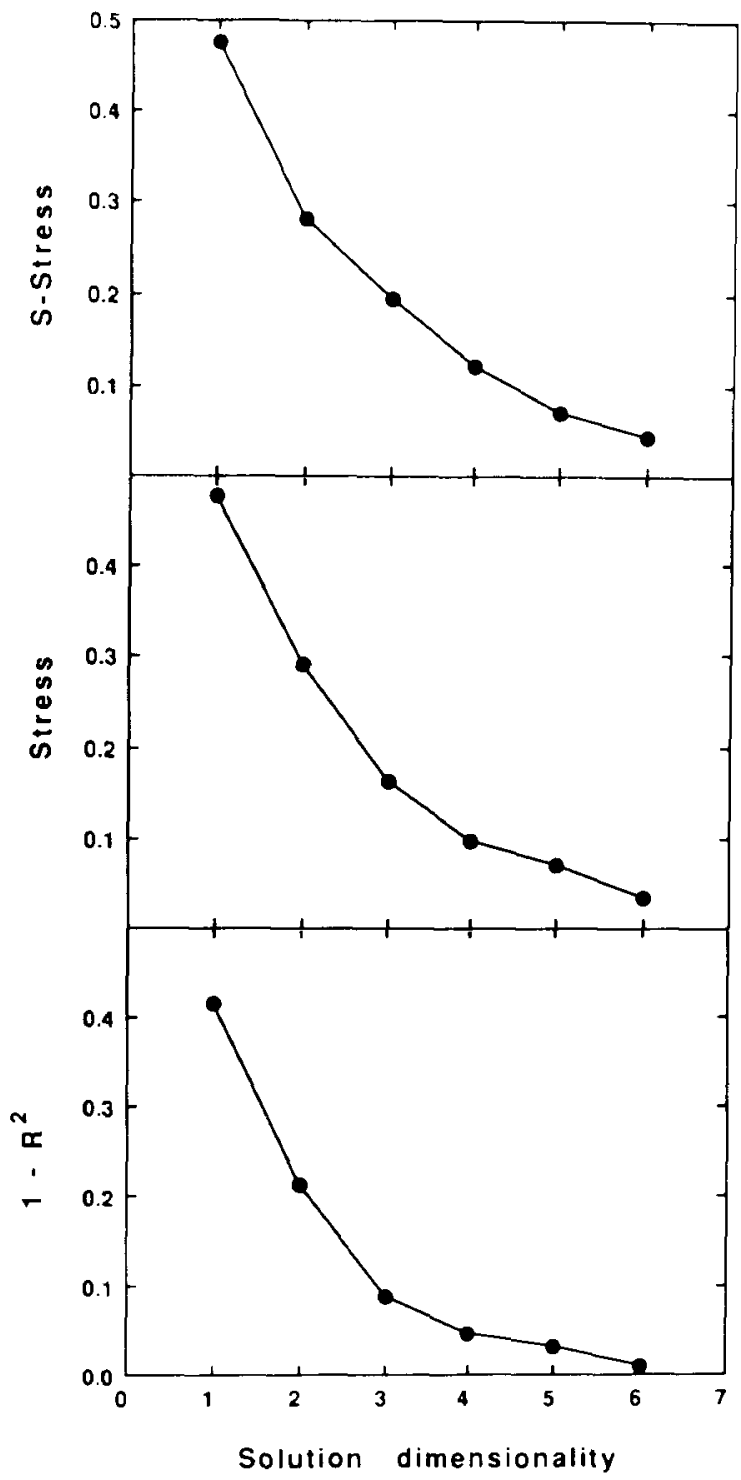

Figure 1. Ability of multidimensional scaling solutions of different dimensionalities to capture information about perceived dissimilarities of the stimuli, as indicated by values of S-Stress (top panel), Stress (center panel), and $1-R^{2}$ (bottom panel).

haps its most salient feature is that it assigns similar values to the plastic and scouring pad (although no subject put them in the same category), while separating them from all other stimuli. While the fourth dimension of the 4dimensional solution is extracting some characteristic of the dissimilarities not emphasized by the 3-dimensional solution, it may be accentuating structure that is idiosyncratic to the present stimulus set: for example, the uniqueness of those few stimuli that were seldom grouped with others may be more fully expressed in the 4-dimensional solution.

In summary, the relative advantages of the higher solution appear minimal, given the fourth dimension's lack of interpretability and the small overall increase in $1-R^{2}$ that it provides.

MDS solution structure. Figure 2 shows the full 3dimensional MDS solution in a rotated orientation, surrounded by a box to enhance depth cuing. The bold-faced parallelogram represents the front of the box (plane of the box closest to the viewer). From the viewer's perspective, the front of the box is tilted slightly down and rotated slightly toward the left. The viewer is also looking directly at the outside surfaces of the "ceiling" and the right "wall" of the box. Values on MDS Solution Dimension 1 increase as one moves from the left toward the right "wall"; values on MDS Solution Dimension 2 increase as one climbs from the box's "floor" toward its "ceiling"; and values on MDS Solution Dimension 3 increase as one moves from the rear "wall" toward the front.

In Figure 2, four primary features of the MDS solution may be distinguished. First, it is clear that in the full MDS solution space, felt and velvet are similar to one another, but different from all other stimuli. In the full space, they lie on the floor of the box, closest to the floor corner nearest the viewer. The straw is also unlike any other stimulus; it lies on the rear wall of the box, about halfway up, and closer to the right wall than to the left. The full 3-dimensional solution space also contains two bands of stimuli. In Figure 2, members of each band are connected by lines. One band, consisting of plastic, wax paper, eraser, tile, cardboard, leather, wood, styrofoam, and cork, begins close to the lower left-hand front corner of the box (where plastic is closest to the viewer), bows outward toward the left wall (in the region of eraser, tile, cardboard, leather, wood, and styrofoam) as it arcs upward, and finally bends back away from the left wall as the band terminates near the ceiling with cork (which is farthest from the viewer). The second band of stimuli contains sandpaper, brick, sponge, bark, and scouring pad. It begins with sandpaper and brick close to the ceiling and nearly against the front of the box, arcs across the upper right-hand corner of the box, and continues with sponge, bark, and scouring pad, which also lie close to the ceiling, but almost against the box's right wall. Scouring pad is located farthest from the viewer.

Examination of the pattern of stimulus points for the 4-dimensional solution (not shown) reveals virtually identical structure and exactly the same four primary characteristics of the space. However, in the 4-dimensional solution, the two bands of points are primarily evident in the Dimension 1-Dimension 4 plane of the solution space. Dimension 2 of the 4-dimensional solution differentiates felt and velvet from the other stimuli, whereas Dimension 3 primarily serves to distinguish straw from all other stimuli. Since felt and velvet (considered as a group) and straw are so different from all other stimuli, the 4dimensional MDS solution achieves its small increment in fit by essentially devoting one dimension exclusively to felt and velvet, and another exclusively to straw. Con- 
Table 2

Scores of the Stimuli on Dimensions of the 3- and 4-Dimensional Multidimensional Scaling Solutions

\begin{tabular}{|c|c|c|c|c|c|c|c|}
\hline \multirow[b]{2}{*}{ Stimulus } & \multicolumn{3}{|c|}{ Dimension } & \multicolumn{4}{|c|}{ Dimension } \\
\hline & 1 & 2 & 3 & 1 & 2 & 3 & 4 \\
\hline Felt & 1.13 & -1.87 & 0.21 & 0.98 & -2.29 & 0.03 & -0.21 \\
\hline Straw & 1.06 & -0.60 & -1.98 & 0.90 & -0.13 & -2.45 & -0.66 \\
\hline Wax paper & -1.18 & -0.39 & 0.38 & -1.32 & -0.14 & 0.32 & 0.64 \\
\hline Cork & -0.95 & 0.43 & -0.99 & -0.90 & 0.17 & -0.27 & -1.44 \\
\hline Tile & -1.25 & 0.08 & -0.04 & -1.33 & 0.11 & 0.34 & -0.43 \\
\hline Cardboard & -1.25 & 0.10 & -0.08 & -1.33 & 0.12 & 0.31 & -0.47 \\
\hline Eraser & -1.29 & -0.02 & 0.18 & -1.41 & 0.20 & 0.30 & 0.33 \\
\hline Styrofoam & -1.21 & 0.14 & -0.61 & -1.34 & 0.38 & -0.66 & -0.25 \\
\hline Brick & 1.42 & 0.87 & 1.09 & 1.81 & 0.58 & 1.13 & -0.47 \\
\hline Plastic & -0.85 & -0.71 & 1.12 & -0.94 & -0.13 & 0.29 & 1.59 \\
\hline Scouring pad & 1.74 & 0.64 & -0.96 & 1.73 & 0.38 & -0.59 & 1.60 \\
\hline Wood & -1.31 & 0.12 & -0.16 & -1.46 & 0.36 & -0.09 & 0.11 \\
\hline Sponge & 1.78 & 0.86 & 0.28 & 2.12 & 0.68 & 0.17 & 0.47 \\
\hline Sandpaper & 1.30 & 1.03 & 1.22 & 1.65 & 0.49 & 1.24 & -1.09 \\
\hline Leather & -1.33 & 0.22 & -0.16 & -1.46 & 0.47 & -0.02 & 0.06 \\
\hline Velvet & 0.41 & -1.82 & 0.54 & 0.22 & -2.18 & 0.34 & 0.11 \\
\hline Bark & 1.76 & 0.92 & -0.04 & 2.07 & 0.91 & -0.37 & 0.09 \\
\hline
\end{tabular}

sequently, medium and large dissimilarities (from pairs containing felt, velvet, or straw) are fit better in the 4dimensional than in the 3-dimensional solution, while small dissimilarities (primarily from pairs not involving felt, velvet, or straw) are left relatively unchanged between the two solutions. Our earlier speculation that the 4-dimensional solution emphasizes local stimulus characteristics appears to be confirmed. We conclude that, given a criterion of parsimony coupled with solution equivalence, the 3-dimensional MDS solution is to be preferred over the 4-dimensional solution.

Interpreting the MDS solution. Adjective-scale ratings were determined by measuring the distance of the subject's vertical mark from the left end of the line on each response slip. For each stimulus, the average rating on each adjective scale was then calculated across subjects. These values, expressed as a proportion of the total length of the scale, are given in Table 4.

The degree of relationship between adjective scales and the 3-dimensional MDS solution space was ascertained by regressing the adjective scales onto the solution space. Each adjective rating scale was used as the dependent variable in a regression analysis with the MDS solution coordinates as the predictor variables. The squared multiple correlation coefficients $\left(R^{2} \mathrm{~s}\right)$ that result from these analyses may be interpreted as the proportion of variance in adjective scales accounted for by the MDS solution- that is, "how much" of each scale lies within the MDS space. The rating scales flat-bumpy and smooth-rough lie almost completely within the MDS space, with $R^{2} s$ of .936 and .960 , respectively. A substantial proportion of the slippery-sticky scale also lies within the MDS space $\left(R^{2}=\right.$ .712 ), while more moderate amounts of the warm-cool
Table 3

Intercorrelations Between Dimensions of the 3- and 4-Dimensional Multidimensional Scaling Solutions

\begin{tabular}{crrr}
\hline & \multicolumn{1}{c}{ Dimension } \\
\cline { 2 - 3 } Dimension & \multicolumn{1}{c}{1} & \multicolumn{1}{c}{ 2 } & \multicolumn{3}{c}{3} \\
\hline 1 & .9941 & .2577 & .0998 \\
2 & -.0361 & .9317 & -.0931 \\
3 & -.0699 & .1776 & .9015 \\
4 & .0491 & -.1363 & .1675 \\
\hline
\end{tabular}

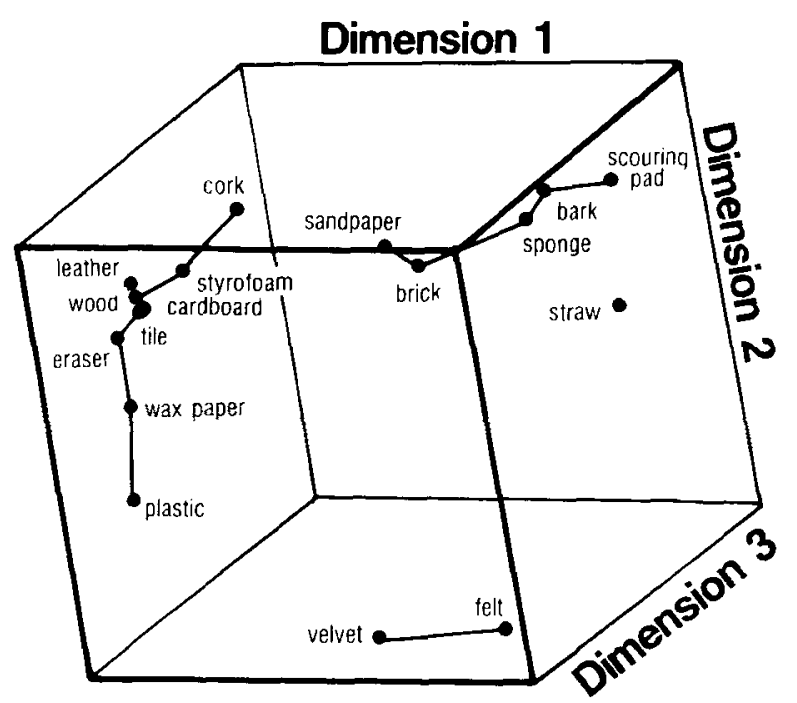

Figure 2. Cube representing the 3-dimensional multidimensional scaling solution. The position of stimulus surfaces in this perceptual space is described in the text. 
and hard-soft scales are subsumed by the MDS space $\left(R^{2} \mathrm{~S}=.521\right.$ and .593 , respectively).

Two-dimensional planes from the 3-dimensional MDS solution are shown in Figure 3. The upper half of the figure represents the view obtained by looking into the solution box (shown in Figure 2) from directly in front; the lower half of Figure 3 shows the view obtained by looking "up" into the box through its floor. The structure of tactile surfaces seen in Figure 2 can also be discerned in Figure 3. In addition, the projections of each adjective rating scale into the MDS space have been added to the display as vectors. Following the suggestions of Shiffman, Reynolds, and Young (1981), the positions of the adjective rating scales in the MDS solution space were determined according to their standardized regression coefficients on each of the three MDS solution dimensions (see Table 5). Standardized regression coefficients were used because sums of squares of standardized regression coefficients are roughly proportional to the variance of the adjective scales "captured" by the MDS space $\left(R^{2} \mathrm{~s}\right)$. (When dimensions of the MDS solution are perfectly uncorrelated, the sum of squares of the standardized regression coefficients will be exactly proportional to the $R^{2}$ s.) Thus, in Figure 3, relatively long vectors are fit better by the display plane than relatively short vectors.

Shown in Table 6 are the correlations, and subtended angles, between the projections of the attribute rating vectors into the 3-dimensional MDS solution space, and the dimensions of the MDS solution. Large positive or negative correlations (or, equivalently, angles close to $0^{\circ}$ or $180^{\circ}$ ) indicate high degrees of relationship between an MDS dimension and an attribute rating scale. If the relationship is large enough, we would be justified in arguing that the MDS dimension is capturing the "same" information about the tactile surfaces as is the rating scale. Examination of Table 6 and Figure 3 reveals notable correlations between the MDS solution's Dimension 1 and

Table 4

Mean Adjective-Scale Ratings Expressed as a Proportion of Distance From First to Second Adjective

\begin{tabular}{lccccc}
\hline \multicolumn{1}{c}{ Stimulus } & $\begin{array}{c}\text { Smooth- } \\
\text { Rough }\end{array}$ & $\begin{array}{c}\text { Hard- } \\
\text { Soft }\end{array}$ & $\begin{array}{c}\text { Slippery- } \\
\text { Sticky }\end{array}$ & $\begin{array}{c}\text { Flat- } \\
\text { Bumpy }\end{array}$ & $\begin{array}{c}\text { Warm- } \\
\text { Cool }\end{array}$ \\
\hline Felt & .49 & .76 & .37 & .40 & .30 \\
Straw & .66 & .17 & .29 & .96 & .48 \\
Wax paper & .18 & .31 & .36 & .11 & .60 \\
Cork & .32 & .26 & .31 & .33 & .49 \\
Tile & .25 & .21 & .24 & .14 & .78 \\
Cardboard & .17 & .29 & .21 & .13 & .59 \\
Eraser & .13 & .31 & .28 & .06 & .71 \\
Styrofoam & .26 & .35 & .40 & .27 & .47 \\
Brick & .76 & .18 & .55 & .65 & .71 \\
Plastic & .15 & .33 & .44 & .07 & .55 \\
Scouring pad & .86 & .57 & .63 & .76 & .32 \\
Wood & .26 & .23 & .40 & .20 & .50 \\
Sponge & .84 & .38 & .57 & .85 & .46 \\
Sandpaper & .85 & .16 & .72 & .40 & .50 \\
Leather & .09 & .31 & .29 & .10 & .63 \\
Velvet & .15 & .83 & .32 & .20 & .34 \\
Bark & .83 & .15 & .45 & .83 & .45 \\
\hline
\end{tabular}

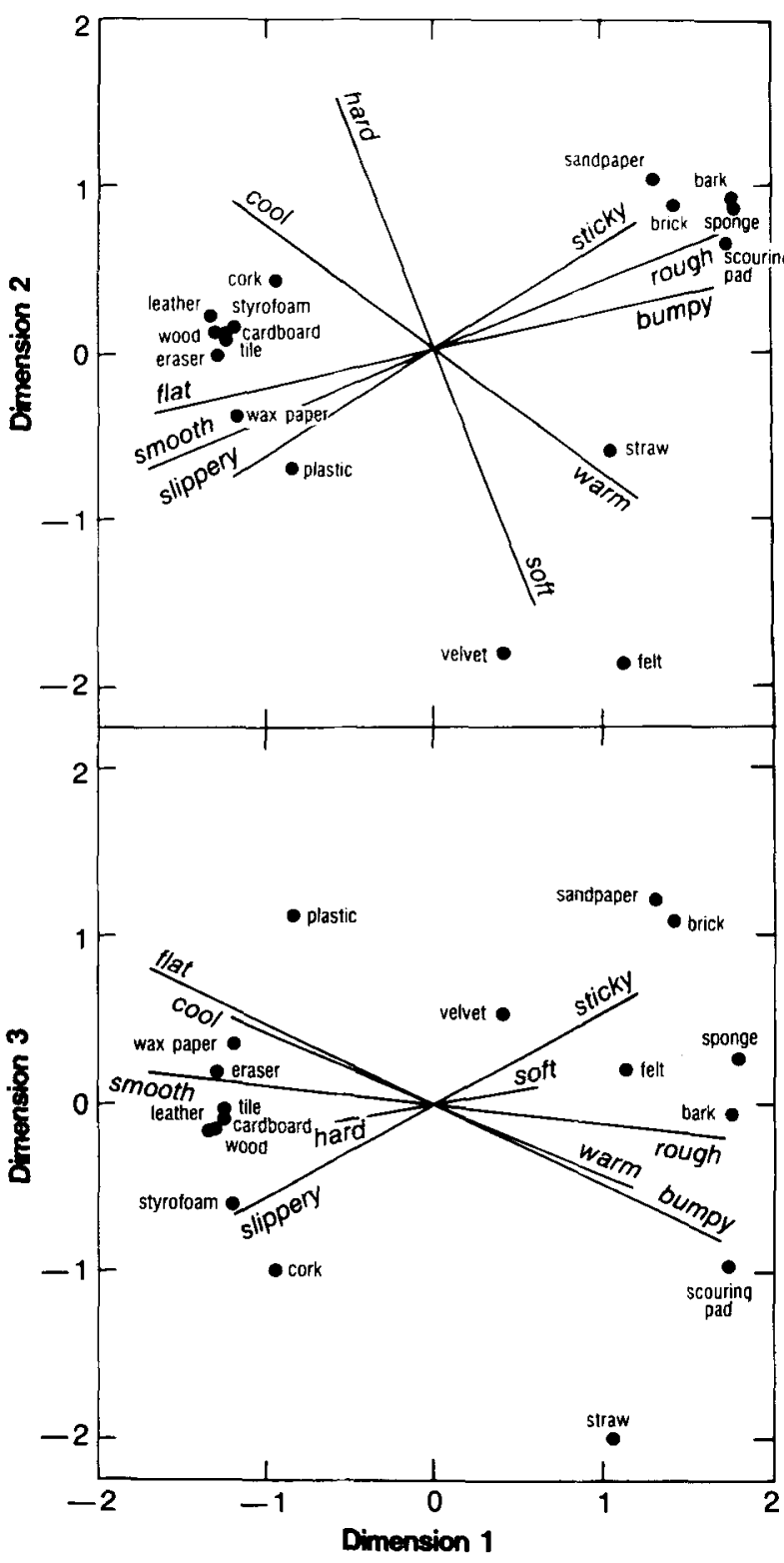

Figure 3. Three-dimensional multidimensional scaling (MDS) solution space, viewed along the third dimension (upper panel) or the second dimension (lower panel). The orientation and relative length of each adjective-scale vector reflect the standardized regression coefficients from regressions of each rating scale onto the MDS soIution space.

the adjective ratings smooth-rough $\left(r=.932, \theta=21^{\circ}\right)$, flat-bumpy $\left(r=.887, \theta=27^{\circ}\right)$, and slippery-sticky $(r=$ $\left..807, \theta=36^{\circ}\right)$. MDS Solution Dimension 2 is highly related to hard-soft ratings $\left(r=-.922, \theta=157^{\circ}\right)$. None of the adjective rating scales appears to capture precisely the same information as does the 3rd MDS solution dimension, as is evidenced by the lack of any dominant correlations. Of the adjective scales employed, Dimension 3 does seem most highly related to slippery-sticky $(r=$ 
Table 5

Standardized Regression Coefficients for Placing

Each Attribute Rating Scale Into the 3-Dimensional Multidimensional Scaling Solution Space

\begin{tabular}{crrrrr}
\multirow{2}{*}{ Dimension } & $\begin{array}{c}\text { S } \begin{array}{c}\text { Smooth- } \\
\text { Rough }\end{array} \\
1\end{array}$ & $\begin{array}{c}\text { Hard- } \\
\text { Soft }\end{array}$ & $\begin{array}{r}\text { Slippery- } \\
\text { Sticky }\end{array}$ & $\begin{array}{r}\text { Flat- } \\
\text { Bumpy }\end{array}$ & \multicolumn{1}{c}{$\begin{array}{c}\text { Warm- } \\
\text { Cool }\end{array}$} \\
\hline 1 & 0.8574 & 0.2947 & 0.5990 & 0.8454 & -0.6063 \\
3 & 0.3479 & -0.7614 & 0.3792 & 0.1911 & 0.4408 \\
3 & -0.0926 & 0.0584 & 0.3307 & -0.4052 & 0.2558 \\
\hline
\end{tabular}

Table 6

Correlations Between Dimensions of the 3-Dimensional Multidimensional Scaling Solution and Adjectival Rating Scales Projected Into the Solution Space

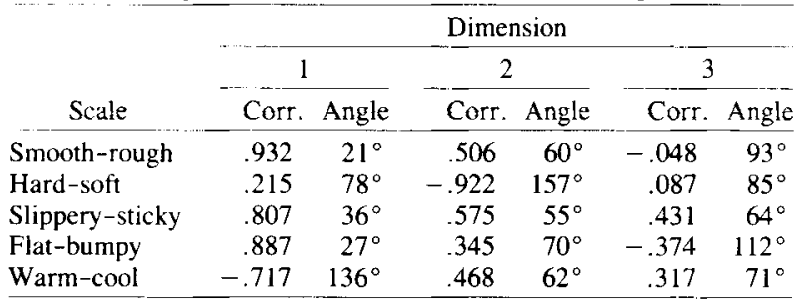

$\left..431, \theta=64^{\circ}\right)$ and to flat-bumpy $(r=-.374, \theta=$ $112^{\circ}$ ); on the other hand, it is unambiguously clear that Dimension 3 is unrelated to both the hard-soft and smoothrough scales. Finally, the warm-cool rating scale appears to cut through all dimensions of the MDS solution space at a moderate angle.

\section{DISCUSSION}

The most important result of this study is its clear demonstration of the fact that subjects' judgments of surface texture can be represented in a perceptual space. The moderate values of stress obtained in the course of MDS analysis suggest that a spatial model is appropriate for conceptualizing sensory differences among the tactile impressions given by the surfaces of everyday objects.

While the dimensionality of perceptual space for texture cannot be determined with certainty, the main features of the present data are reasonably well captured by a 3-dimensional solution. On the basis of the goodnessof-fit measures (Stress, S-Stress, and $1-R^{2}$ ) and on the nature (i.e., characteristics and appearances) of the solutions themselves, it is clear that the 1- and 2-dimensional solutions are inadequate. Although some features of the perceptual space examined here may be specific to the stimulus textures employed, and it is possible that further studies using different stimulus sets would demonstrate the existence of additional dimensions, we consider it unlikely that there are fewer than 3 dimensions.

It is more difficult to rule out the possibility that there are more than 3 dimensions. A close comparison of the 3- and 4-dimensional solutions, however, showed that the latter produced its small improvement in $1-R^{2}$ and related measures, largely by allowing for fuller-indeed, dimensional-expression of the uniqueness of a small number of stimuli perceived as very different from the others. It is argued here that this feature of the 4dimensional solution is not so advantageous as to outweigh the parsimony of retaining the 3-dimensional solution. The same argument applies with even greater force to solutions with dimensionalities greater than 4 . On the other hand, it may be that different stimuli, or different conditions of stimulation (allowing free exploration of surfaces, for example), would establish the existence of additional perceptual dimensions of tactile surface texture. The conditions of stimulation are likely to be particularly important, given the demonstration by Lederman and Klatzky (1987) that the "exploratory procedures" employed by subjects play a major role in determining what information will be extracted. Further research will be needed to test the effects of these factors on the dimensionality of perceived surface texture.

The adjective scales in this study were all found to allow subjects to describe their tactile perceptions, at least to some extent. Each scale, when optimally positioned in the 3-dimensional MDS model, correlated with the 3dimensional MDS solution to a substantial degree. The compatibility of these two sets of data with one another indicates that subjects' perceptions of surface texture are well organized, robust, and flexible enough for subjects to be able to convey information about them in more than one way.

Regarding the identity of the perceptual dimensions themselves, the high $R^{2}$ obtained for the smooth-rough dimension suggests its primacy as a descriptor of surface textures. This established, the hard-soft dimension becomes a likely perceptual dimension, because it is essentially perpendicular to the rough-smooth dimension in MDS space (see Figure 3); this is not true for any of the other adjective scales employed in this study.

The flat-bumpy scale was intended to allow subjects the opportunity to rate stimuli on large surface features, as distinct from the microtextural features that are captured by the terms smooth and rough. The data did not fulfill this expectation, however: Flat-bumpy ratings were closely correlated with smooth-rough ratings ( $r=.891$ ), and the vectors representing these two scales had similar $R^{2}$ values (.936 and .960 , respectively) with respect to the MDS space. Subjects apparently used the two scales as roughly synonymous.

Despite the attractive features of the 3-dimensional MDS solution, then, only 2 perceptual dimensions-those frequently mentioned in the literature - were readily identifiable. The fact that warm-cool ratings were not independent of those for roughness and hardness suggests that, while some stimuli felt colder than others, temperature sensations alone were not responsible for the 3dimensionality of the perceptual space. Likewise, sensations of slipperiness and stickiness may have contributed to, but did not themselves constitute, the 3rd dimension.

The stimulus farthest "down" on the 3rd dimensionfarthest below the plane formed by the roughness and hardness axes in Figure 3-is woven straw; next lower 
are the cork, scouring pad, and styrofoam. One thing that all four of these surfaces have in common is a high compressional elasticity, a springiness that causes them to bounce back when compressed and released. The three stimuli that are at the other "end" of Dimension 3 are the sandpaper, plastic, and brick-surfaces that are very much lacking in this quality. "Springiness," then, is one possible interpretation of the 3rd MDS dimension. Although the locations of some of the stimuli raise problems for this idea, it seems worthy of further and more explicit testing-for example, by including "springiness" among the qualities to be rated in future studies.

In summary, the data from the adjective scales show that under the conditions of stimulus presentation used in this study, subjective dissimilarities among surface textures, captured by the MDS analysis, can be described to a large extent by dimensions on which any tangible surface can be rated. This suggests that the characteristic "feel" of particular objects-Katz's Spezifikationendepends on particular combinations of dimensional values, rather than on unique, dimensionless properties.

\section{REFERENCES}

CATTELL, R. B. (1978). The scientific use of factor analysis in behavioral and life sciences. New York: Plenum.

Connor, C. E., Hsiao, S. S., Phillups, J. R., \& Johnson, K. O. (1990). Tactile roughness: Neural codes that account for psychophysical magnitude estimates. Journal of Neuroscience, 10, 3823-3836.

Ekman, G., Hosman, J., \& LinDSTrom, B. (1965). Roughness, smoothness, and preference: A study of quantitative relations in individual subjects. Journal of Experimental Psychology, 70, 18-26.

GREEN, B. G. (1981). Tactile roughness and the "paper effect." Bulletin of the Psychonomic Society, 18, 155-158.

KATZ, D. (1989). The world of touch (L. E. Krueger, Trans.). Hillsdale, NJ: Erlbaum. (Original work published 1925)

Klatzky, R. L., Lederman, S., \& Reed, C. (1987). There's more to touch than meets the eye: The salience of object attributes for hap tics with and without vision. Joumal of Experimental Psychology: Gen eral, 116, 356-369

Klatzky, R. L., Lederman, S. J., \& Reed, C. (1989). Haptic integration of object properties: Texture, hardness, and planar contour. Jour nal of Experimental Psychology: Human Perception \& Performance. $15,45-57$.

KrUSKAL, J. B. (1964a). Multidimensional scaling by optimizing goodness of fit to a nonmetric hypothesis. Psychometrika, 29, 1-27.

KruSKal, J. B. (1964b). Nonmetric multidimensional scaling: A numerical method. Psychometrika, 29, 115-129.

KrUSKAL, J. B., \& WISH, M. (1978). Multidimensional scaling. Beverly Hills, CA: Sage.

Lederman, S. J. (1978a). Heightening tactile impressions of surface texture. In G. Gordon (Ed.), Active touch: The mechanism of recognition of objects by manipulation. A multi-disciplinary approach (pp. 205-214). Oxford, U.K.: Pergamon.

LedERMan, S. J. (1978b). "Improving one's touch" ... and more Perception \& Psychophysics, 24, 154-160.

Lederman, S. J., \& KLATZKY, R. L. (1987). Hand movements: A window into haptic object recognition. Cognitive Psychology, 19, 342-368.

Lederman, S. J., \& TAYLOR, M. M. (1972). Fingertip force, surface geometry, and the perception of roughness by active touch. Perception \& Psychophysics, 12, 401-408.

Shiffman, S. S., Reynolds, M. L., \& Young, F. W. (1981). Handbook of multidimensional scaling. New York: Academic Press.

STEvens, S. S., \& HarRIS, J. R. (1962). The scaling of subjective roughness and smoothness. Journal of Experimental Psychology, 64, 489-494.

Takane, Y., Young, F. W., \& De Leeuw, J. (1977). Nonmetric individual differences multidimensional scaling: An alternating least squares method with optimal scaling features. Psychometrika, 41, 505-529.

TAylor, M. M., \& Lederman, S. J. (1975). Tactile roughness of grooved surfaces: A model and the effect of friction. Perception \& Psychophysics, 17, 23-36.

Young, F. W., \& LEWyCKY, R. (1979). ALSCAL-4 user's guide (2nd ed.). Carrboro, NC: Data Analysis and Theory Associates.

(Manuscript received March 2, 1992; revision accepted for publication June 1, 1993.) 